\title{
COMPOSIÇÃO DO BANCO DE SEMENTES NO SOLO EM ÁREAS DE PRESERVAÇÃO PERMANENTE SOB DIFERENTES TIPOS DE COBERTURA
}

\author{
Lauri Amândio Schorn ${ }^{1}$, Tatiele Anete Bergamo Fenillii ${ }^{2}$, Andres Krüger ${ }^{3}$, Gabriel Corso Pellens ${ }^{4}$, \\ Juliana Jaqueline Budag ${ }^{5}$, Marmonn Canestraro Nadolny ${ }^{6}$ \\ ${ }^{1}$ Eng. Florestal, Dr., Depto. de Engenharia Florestal, FURB, Blumenau, SC, Brasil - lauri.schorn@ gmail.com \\ ${ }^{2}$ Eng $^{\mathrm{a}}$ Agrônoma, Dr ${ }^{\mathrm{a}}$., Depto. de Engenharia Florestal, FURB, Blumenau, SC, Brasil - tfenilli@ furb.br \\ ${ }^{3}$ Eng. Florestal, Depto. de Engenharia Florestal, FURB, Blumenau, SC, Brasil - andres_kruger@yahoo.com.br \\ ${ }^{4}$ Eng. Florestal, Mestrando em Engenharia Florestal, FURB, Blumenau, SC, Brasil - gabrielpellens@ @erra.com.br \\ ${ }^{5}$ Acadêmica de Engenharia Florestal, FURB, Blumenau, SC, Brasil - julianabudag@ gmail.com \\ ${ }^{6}$ Eng. Florestal, M.Sc.,Valor Florestal, Rio Negrinho, SC, Brasil - valorflorestal@ matrix.com.br \\ Recebido para publicação: 09/05/2011 - Aceito para publicação: 15/02/2013
}

\begin{abstract}
Resumo
Este estudo teve como objetivo avaliar a composição e similaridade do banco de sementes no solo em três áreas com diferentes históricos de cobertura do solo: I - floresta nativa remanescente; II - extração recente de Pinus; III - reflorestamento de Pinus. Foram coletadas 10 amostras de solo aleatoriamente, para cada uma das três áreas. As amostras de solo foram colocadas em bandejas de $60 \times 40 \times 10 \mathrm{~cm} \mathrm{e}$ dispostas em canteiros a céu aberto no laboratório de silvicultura da FURB, em Blumenau, SC, para análise da germinação, entre junho de 2008 e maio de 2009. As plântulas germinadas foram classificadas em grupos: arbóreas, arbustivas, cipós, herbáceas, gramíneas, pteridófitas e arbóreas exóticas. Foram identificados 2223 indivíduos, pertencentes a 37 espécies e 22 famílias. As espécies com maior quantidade de sementes germinadas nos três ambientes foram Eupatorium compressum e Braquiaria subquadripara. A densidade de indivíduos $/ \mathrm{m}^{2}$ foi de 2.846 (ambiente I), 4.292 (ambiente II) e 2.125 (ambiente III). O número de espécies, diversidade e equabilidade foram decrescentes do ambiente I para o ambiente III. Concluiu-se que o tipo de uso e cobertura da área tem relação com a densidade dos grupos de plantas presentes no banco de sementes no solo.

Palavras-chave: Regeneração; Floresta Ombrófila Mista; recuperação de área.
\end{abstract}

\begin{abstract}
Composition of soil seed bank in areas of permanent preservation under different types of coverage. The aim of this study was to evaluate the composition of soil seed bank in three types of use of permanent preservation area: I - remaining native Forest; II - recent extraction of Pinus; III reforestation of Pinus. 10 soil samples were collected in randomly points distributed in each one of the three areas. The soil samples were individually placed in plastic trays with $60 \mathrm{x} 40 \mathrm{x} 10 \mathrm{~cm}$ and arranged in open plots in the Silviculture Laboratory of FURB in Blumenau - SC, for analysis of germination, from June 2008 to May 2009. The seedlings were classified into groups: trees, shrubs, vines, herbs, grasses, ferns and pine. 2223 individuals were identified; they belonged to 37 species and 22 families. The species with the largest number of germinated seeds within the three environments were Eupatorium compressum and Braquiaria subquadripara. The density was 2846 indivíduals.m ${ }^{2}$ (environment I), 4292 (environment II) and 2125 (environment III). The number of species and diversity values decreased from environment with native remaining forest to the environment area with Pinus. Results point to the fact that the type of use and coverage of the area is related to density of plant groups present in the soil seed bank.
\end{abstract}

Keywords: Regeneration; Mixed Forest; reclamation area.

\section{INTRODUÇÃO}

Áreas consideradas de preservação permanente, de acordo com a legislação atual, foram ocupadas por reflorestamentos ou atividades agropecuárias em décadas passadas, e atualmente, para se 
adequar à legislação ambiental, faz-se necessária a recuperação da vegetação natural nessas áreas. No entanto, a realização da recuperação dessas áreas de forma eficiente ainda é dependente de novas informações científicas relacionadas aos processos de recuperação.

Nesse sentido, Schmitz (1992) afirmou que a recolonização pela vegetação em um ambiente perturbado ocorre principalmente através dos bancos de sementes no solo, mantendo este um papel fundamental no equilíbrio dinâmico da floresta.

Denomina-se banco de sementes no solo a todas as sementes viáveis no solo ou associadas à serapilheira, para uma determinada área num dado momento. É um sistema dinâmico, com entrada de sementes através da chuva de sementes, e dispersão, podendo ser transitório, com sementes que germinam dentro de um ano após o início da dispersão, ou persistente, com sementes que permanecem no solo por mais de um ano. Essa persistência personifica, segundo Simpson et al. (1989), uma reserva do potencial genético acumulado.

Além disso, o banco de sementes do solo reflete a composição potencial da floresta após perturbações (BAIDER et al., 1999). É um dos fatores mais importantes na recolonização natural de áreas perturbadas, dando início ao processo sucessional (VIEIRA et al., 2003), representando um depósito de elevada densidade, com ampla variedade de espécies em estado de latência, sobretudo de espécies pioneiras e secundárias iniciais (UHL; CLARK., 1983; GARWOOD, 1989; PINÃ-RODRIGUES et al., 1990).

O estoque de sementes no solo é formado por espécies representativas da vegetação atual, espécies de etapas sucessionais anteriores e espécies nunca antes presentes na área, mas que formam parte do banco (SORREANO, 2002). Tem como fonte as chuvas de sementes, que pode provir da comunidade local, da vizinhança ou de áreas distantes, quando ocorre o processo de dispersão (HALL; SWAINE, 1980). As sementes produzidas no local promovem a autorregeneração da floresta, enquanto aquelas que são trazidas por agentes dispersores representam o avanço da regeneração de indivíduos e espécies externas da área (MARTINEZ-RAMOS; SOTO-CASTRO, 1993).

A densidade de sementes no solo pode deferir com a região, o histórico da área, o tipo de vegetação e a profundidade de coleta (GARWOOD, 1989), sendo observada uma maior proporção de sementes nos primeiros $5 \mathrm{~cm}$ do solo (PUTZ, 1983). Já o período de permanência das sementes no banco é determinado pelas propriedades fisiológicas, tais como germinação, dormência e viabilidade (OLIVEIRA, 1993), condições ambientais, como mudanças térmicas ou luminosas, provocadas após aberturas de clareiras (BROKAW, 1985; WHITMORE, 1978; 1996) e pela presença de predadores e patógenos de sementes no ambiente (PARKER, 1989).

Alguns autores (HARPER, 1977; UHL et al., 1988; GARWOOD, 1989; BAIDER et al., 1999) também citaram que o banco de sementes em florestas tropicais está envolvido em, pelo menos, quatro processos nos níveis de população e de comunidade: o estabelecimento de populações, a manutenção da diversidade de espécies, o estabelecimento de grupos ecológicos e a restauração da riqueza de espécies durante a regeneração da floresta após distúrbios naturais ou antrópicos.

Os elevados valores de densidade e riqueza encontrados no banco do solo na maioria dos levantamentos realizados em florestas brasileiras (MARTINS et al., 2009 ) evidenciam o potencial de utilização desse banco na restauração florestal em áreas degradadas, contribuindo para o aumento da diversidade e a redução dos custos de implantação e manutenção dos projetos de restauração (MARTINS, 2009).

Em áreas de preservação permanente ocupadas por reflorestamentos, em geral observa-se uma vegetação de sub-bosque que pode ser rala, no caso de povoamentos de Pinus, ou densa, quando sob povoamentos de Eucalyptus. Após a extração dos povoamentos e caso haja abandono da área, observa-se o aumento gradativo da densidade e riqueza dessa vegetação, em função da germinação de sementes no banco presente no solo ou do ingresso de propágulos de áreas adjacentes. Podem também contribuir para esse ingresso de propágulos os fragmentos de florestas nativas remanescentes nas áreas de preservação permanente.

Diante do exposto, o presente trabalho teve por objetivo avaliar a composição e similaridade do banco de sementes no solo em três áreas com diferentes históricos de cobertura do solo: I - com Floresta Ombrófila Mista ciliar remanescente; II - com extração recente de povoamento de Pinus taeda Lineu.; III - com reflorestamento de Pinus taeda.

\section{MATERIAL E MÉTODOS}

O presente trabalho foi realizado em uma área de produção de Pinus taeda da empresa Renova 
Floresta, no município de Rio Negrinho, SC, situada nas coordenadas $26^{\circ} 11^{\prime} 33^{\prime}$ ' de latitude sul e 49³2'15” de longitude oeste do Meridiano de Greenwich (Figura 1).

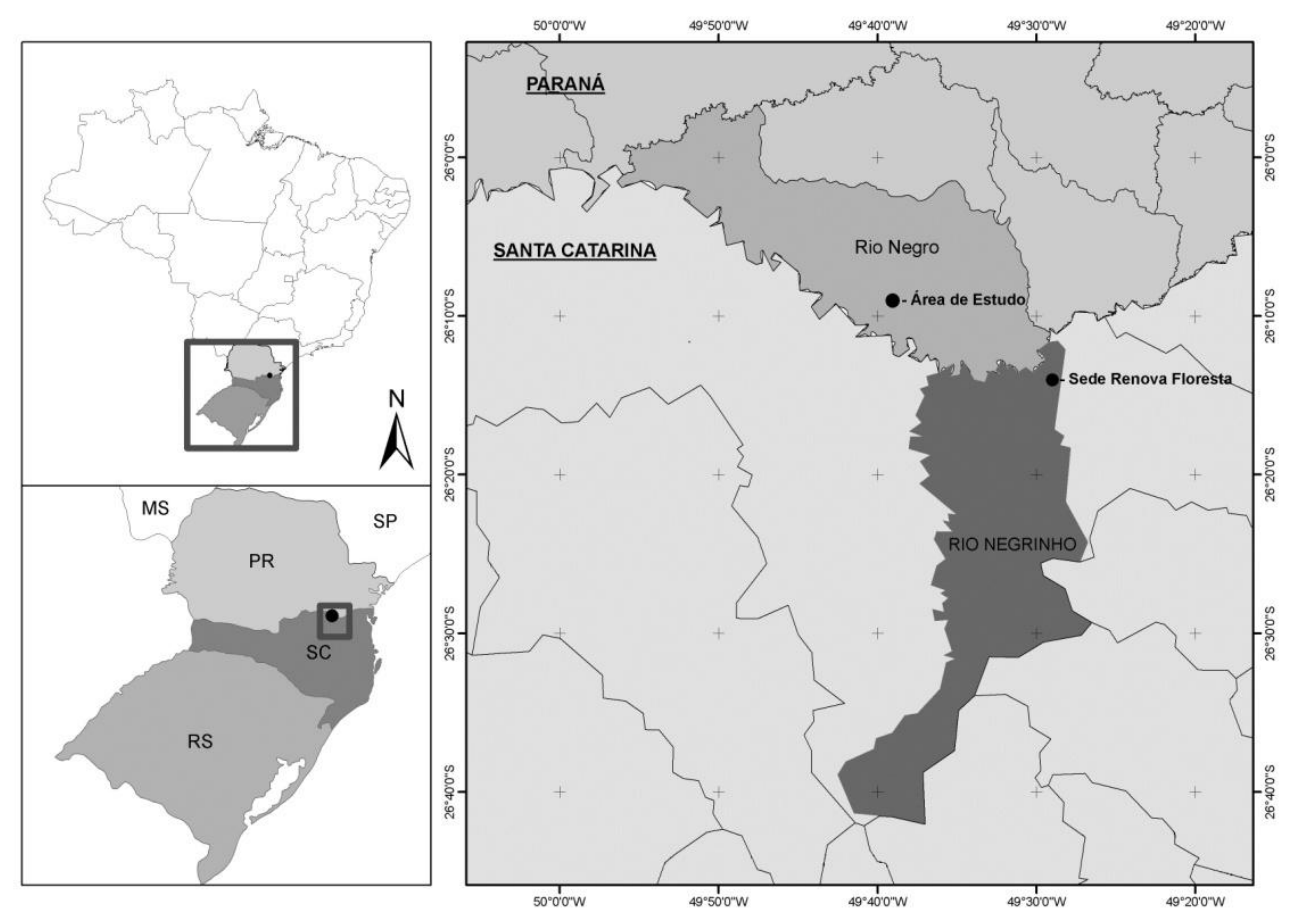

Figura 1. Localização do município de Rio Negrinho, região norte de Santa Catarina.

Figure 1. Localization of Rio Negrinho, north region of Santa Catarina.

A altitude no local é de aproximadamente 841 metros. O clima na região é caracterizado como $\mathrm{Cfb}$ : mesotérmico, subtropical úmido, com verões frescos, sem estação seca e com geadas severas frequentes, segundo a classificação de Köppen (GAPLAN, 1986). No âmbito geológico, a região é constituída de rochas sedimentares paleozoicas (KOBIYAMA et al., 2007). O relevo regional é suave ondulado a ondulado.

Os solos da área estudada são classificados como Cambissolo Háplico Alítico, que variam de rasos a profundos, possuindo sequência de horizonte $\mathrm{A}, \mathrm{Bi}, \mathrm{C}$, com diferenciação de horizontes visualmente modesta. O horizonte B apresenta cores amareladas e brunadas, com matiz de 5 a $10 \mathrm{YR}$. A análise química do solo na área estudada apresentou os seguintes parâmetros: textura $29 \%$ de argila; $\mathrm{pH} 3,80$; matéria orgânica $6,00 \%$; fósforo $5,50 \mathrm{ppm}$; potássio $32,00 \mathrm{ppm}$; alumínio $7,30 \mathrm{cmol}_{\mathrm{c}} /$; cálcio 0,80 $\mathrm{cmol}_{\mathrm{c}} / \mathrm{l}$; magnésio $0,40 \mathrm{cmol}_{\mathrm{c}} \mathrm{l} ; \mathrm{H}+\mathrm{Al} 43,35 \mathrm{cmol} / 1$; CTC 44,64 cmol/l; e saturação por bases $2,89 \%$. A profundidade do solo na área apresenta variações de 1,0 a 1,5 metros.

A coleta de amostras de solo foi realizada em uma única vez, no mês de julho de 2008, em três ambientes, localizados em áreas de preservação permanente: com floresta nativa remanescente (ambiente I), com extração de povoamento de Pinus três meses antes do início do estudo (ambiente II) e com reflorestamento remanescente de Pinus (ambiente III).

Os ambientes estavam inseridos e situados de forma adjacente em um talhão de reflorestamento de Pinus taeda, cuja área abrangia aproximadamente 5,0 hectares. A coleta das amostras de solo foi realizada em pontos distribuídos de forma aleatória em cada um dos ambientes, tendo uma distância mínima de 30 metros entre as amostras. Os pontos de coleta consistiram de parcela medindo 1 x $0,30 \mathrm{~m}$ e profundidade de $10 \mathrm{~cm}$. Em cada ambiente (tratamento) foram coletadas 10 amostras, que constituíram as repetições. O solo foi coletado utilizando-se uma pá, armazenado em sacos plásticos, identificados por etiquetas, e transportados para o viveiro florestal e laboratório de silvicultura da Universidade Regional de Blumenau (FURB).

No viveiro, as amostras foram colocadas em bandejas plásticas de $60 \times 40 \times 12 \mathrm{~cm}$ de dimensões, com 4 furos no fundo. Sobre a superfície de cada amostra, foi depositada uma camada de 
$3 \mathrm{~cm}$ de vermiculita. Posteriormente, as bandejas foram dispostas em canteiros ao ar livre.

A avaliação do experimento consistiu em quantificar o número de plântulas que emergiram das amostras de solo. As avaliações foram iniciadas em julho de 2008 e realizadas mensalmente, até maio de 2009.

As plântulas identificadas foram contadas e retiradas das bandejas no momento de cada avaliação. Plântulas não identificadas foram transplantadas para substratos em sacos de polietileno, para identificação posterior, em fase de maior desenvolvimento. A identificação das espécies germinadas foi realizada no laboratório de dendrologia da FURB e através de consulta a especialistas.

As espécies encontradas foram classificadas em sete grupos: arbóreas, arbustivas, cipós, herbáceas, gramíneas, pteridófitas e arbóreas exóticas. A determinação dos sete grupos foi realizada de forma a abranger os grupos de espécies mais frequentes em áreas adjacentes, de acordo com observações realizadas previamente. A inclusão de arbóreas exóticas como um grupo foi realizado para distinguir os indivíduos de Pinus das espécies arbóreas de ocorrência natural.

Para cada grupo de espécies, foram analisadas a densidade de plântulas germinadas, a riqueza específica, a diversidade, através do índice de Shannon, e a equabilidade, através do índice de Pielou (BROWER; ZAR, 1984).

Foi realizada também análise de variância entre as médias das densidades dos tratamentos, pelo teste de Tukey ao nível de 5\% de probabilidade. Previamente à realização dessas análises, efetuou-se o teste de Shapiro-Wilks, para avaliar a normalidade dos dados. quadrados.

A quantidade total de indivíduos germinados por bandeja foi transformada para metros

\section{RESULTADOS E DISCUSSÃO}

No levantamento das sementes germinadas nas amostras de solo dos três ambientes, foram identificados 2223 indivíduos, pertencentes a 37 espécies e 22 famílias, distribuídos nos grupos, arbóreas, arbustivas, cipós, herbáceas, gramíneas, pteridófitas e Pinus (Tabela 1).

No ambiente I, emergiram 30 espécies, distribuídas em 13 famílias, sendo as mais expressivas em número de plântulas Asteraceae $(28,1 \%)$ e Poaceae $(17,7 \%)$. No ambiente II, foram observadas 28 espécies germinadas, distribuídas em 16 famílias, sendo que as famílias Asteraceae $(22,5 \%)$ e Poaceae $(19,1 \%)$ foram as mais representativas também. No ambiente III, foram identificadas 25 espécies, distribuídas em 16 famílias, representadas principalmente por Asteraceae (17,7\%) e Cyperaceae $(20,2 \%)$.

A riqueza florística encontrada nos três ambientes situa-se entre os valores apontados por Garwood (1989), que citou entre 8 e 67 espécies para ambientes alterados em florestas tropicais, mas foi inferior aos valores obtidos por Araújo et al. (2001) e por Silva (2008).

As famílias Asteraceae e Cyperaceae apresentaram o maior número de plântulas emergidas e o maior número de espécies nos três ambientes estudados. Estudos realizados por Souza (1996) também apontaram que as espécies com maior quantidade de sementes pertenceram às famílias Asteraceae e Cyperaceae. Chami (2008) também encontrou resultados semelhantes, em que as famílias com maior número de indivíduos no banco de sementes foram Asteraceae, Commelinaceae e Cyperaceae.

Os números totais de indivíduos germinados foram distintos entre os três ambientes estudados. No banco de sementes da área com extração recente de reflorestamento, foi observada a maior densidade, com 4292 indivíduos $/ \mathrm{m}^{2}$, enquanto na área sob reflorestamento o banco de sementes apresentou a menor densidade, com 2125 indivíduos $/ \mathrm{m}^{2}$ (Tabela 2). No entanto, o resultado da análise da variância demonstrou que não houve diferença significativa no número de indivíduos $/ \mathrm{m}^{2}$ entre os ambientes. Esse fato mostra que há uma heterogeneidade elevada na distribuição espacial da densidade de sementes no solo e que esse padrão é semelhante nos três ambientes.

O número de espécies, a diversidade e a equabilidade foram decrescentes do ambiente I para o ambiente III, evidenciando o potencial dos fragmentos naturais remanescentes como indutores da regeneração natural em áreas adjacentes. Por outro lado, a baixa diferença entre as médias dessas variáveis para os três ambientes revela que solos sob povoamentos de Pinus podem abrigar um banco de sementes com relativo potencial para iniciar os processos de recuperação da vegetação natural nessas áreas. 
Tabela 1. Distribuição das espécies por grupos no banco de sementes do solo em áreas de preservação permanente na região de Rio Negrinho, SC. ARV: arbóreas; ARBU: arbustivas; CIPÓ: cipós; ERVA: ervas; GRAM: gramíneas; PTE: pteridófitas; AE: arbóreas exóticas. Ambiente I: área sob floresta nativa remanescente; Ambiente II: área onde ocorreu extração de povoamento de Pinus taeda; Ambiente III: área sob povoamento de Pinus taeda.

Table 1. Distribution of species groups in soil seed bank in the permanent preservation areas in the region of Rio Negrinho, SC. ARV: tree; ARBU: shrubs; CIPÓ: vine; ERVA: herbs; GRAM: grass; PTE: pteridophyte; AE: exotic trees.

\begin{tabular}{|c|c|c|c|c|c|c|c|}
\hline \multirow{2}{*}{ Família/espécie } & \multirow{2}{*}{ Nome vulgar } & \multicolumn{6}{|c|}{ Formas de vida } \\
\hline & & ARV & ARBU & ERVA & CIPÓ & GRAM & PTE PN \\
\hline \multicolumn{8}{|l|}{ AQUIFILIACEAE } \\
\hline Ilex paraguariensis & Erva-mate & $\mathrm{x}$ & & & & & \\
\hline \multicolumn{8}{|l|}{ ASTERACEAE } \\
\hline Baccharis trimera & Carqueja & & & $\mathrm{x}$ & & & \\
\hline Baccharis uncinella & Óleo-de-vassoura & & $\mathrm{x}$ & & & & \\
\hline Conzia canadensis & Voadeira & & & $\mathrm{x}$ & & & \\
\hline Erechtites valerianifolius & Capiçoba & & & $\mathrm{x}$ & & & \\
\hline Eupatorium compressum & Eupatório & & $\mathrm{x}$ & & & & \\
\hline Gamochaeta sp. & Espiga-de-ouro & & & $\mathrm{x}$ & & & \\
\hline Gnaphalium pensylvanicum & Marcela & & & $\mathrm{x}$ & & & \\
\hline Louteigia ballotifolia & Pixiricão-roxo & & & $\mathrm{x}$ & & & \\
\hline Piptocarpha angustifolia & Vassourão-branco & $\mathrm{x}$ & & & & & \\
\hline Siegesbekia orientalis & Botão-de-ouro & & & $\mathrm{x}$ & & & \\
\hline Venonantura sp. & Vassourinha & & & $\mathrm{x}$ & & & \\
\hline \multicolumn{8}{|l|}{ COMMELINACEAE } \\
\hline Commelina diffusa & Grama-da-terra & & & & & $\mathrm{x}$ & \\
\hline Commelina erecta & Trapoereba & & & & & $\mathrm{x}$ & \\
\hline \multicolumn{8}{|l|}{ CONVOLVULACEAE } \\
\hline Ipomea nil & Corda-de-viola & & & & $\mathrm{x}$ & & \\
\hline CYPERACEAE & & & & & & $\mathrm{x}$ & \\
\hline Bulbostylis capillaris & Alecrim-da-praia & & & & & $\mathrm{x}$ & \\
\hline Cyperus meyenianus & Tiririca & & & & & & \\
\hline \multicolumn{8}{|l|}{ EUPHORBIACEAE } \\
\hline Croton urucurana & Sangueiro & $\mathrm{x}$ & & & & & \\
\hline Sebastiana commersoniana & Branquinho & $\mathrm{x}$ & & & & & \\
\hline \multicolumn{8}{|l|}{ FABACEAE } \\
\hline Collea speciosa & Colea & & $\mathrm{x}$ & & & & \\
\hline \multirow{2}{*}{\multicolumn{8}{|c|}{ IRIDACEAE }} \\
\hline & & & & & & & \\
\hline Sisyrinchium sp. & Cebolinha & & & & & $\mathrm{x}$ & \\
\hline \multicolumn{8}{|l|}{ LAMINACEAE } \\
\hline Aegiphila sellowiana & Gaioleira & $\mathrm{x}$ & & & & & \\
\hline \multicolumn{8}{|l|}{ LAURACEAE } \\
\hline Cinпатотит атоепит & Canela-pimenta & & $\mathrm{x}$ & & & & \\
\hline Ocotea puberula & Canela-guaicá & $\mathrm{x}$ & & & & & \\
\hline \multicolumn{8}{|l|}{ MALVACEAE } \\
\hline Sida sp. & Guanxumba & & & $\mathrm{x}$ & & & \\
\hline \multicolumn{8}{|l|}{ MYRSINACEAE } \\
\hline Myrsine coriacea & Capororoca & & $\mathrm{x}$ & & & & \\
\hline \multicolumn{8}{|l|}{ PHYLLANTHACEAE } \\
\hline Phyllanthus niruri & Quebra-pedra & & & $\mathrm{x}$ & & & \\
\hline \multicolumn{8}{|l|}{ PHYTOLACACEAE } \\
\hline Phytolacca americana & Caruru & & & $\mathrm{x}$ & & & \\
\hline
\end{tabular}




\begin{tabular}{|c|c|c|c|c|c|c|}
\hline \multicolumn{7}{|l|}{ PINACEAE } \\
\hline Pinus sp. & Pínus & & & & & $\mathrm{x}$ \\
\hline \multicolumn{7}{|l|}{ PLANTAGINACEAE } \\
\hline Scoparia dulcis & Vassourinha-doce & & $\mathrm{x}$ & & & \\
\hline \multicolumn{7}{|l|}{ POACEAE } \\
\hline Axonopus fissifolius & Grama & & & $\mathrm{x}$ & & \\
\hline Braquiaria sebastiana & Papanduva & & & $\mathrm{x}$ & & \\
\hline Digitaria ciliares & Capim-colchão & & & $\mathrm{x}$ & & \\
\hline \multicolumn{7}{|l|}{ POLYPODIACEAE } \\
\hline Polypodium sp. & Samambaia & & & & $\mathrm{x}$ & \\
\hline \multicolumn{7}{|l|}{ RUBIACEAE } \\
\hline Mitracarpus hirtus & Poaia-da-praia & & $\mathrm{x}$ & & & \\
\hline Mitracarpus villosus & Erva-quente & & $\mathrm{x}$ & & & \\
\hline \multicolumn{7}{|l|}{ SOLANACEAE } \\
\hline Solanum variabile & Jurubeba-velame & $\mathrm{x}$ & & & & \\
\hline Solanum verbascifolium & Fumo-bravo & $\mathrm{x}$ & & & & \\
\hline
\end{tabular}

Tabela 2. Valores das variáveis de densidade e diversidade nas amostras do banco de sementes do solo de três ambientes em áreas de preservação permanente na região de Rio Negrinho, SC.

Table 2. Values of variable density and diversity in the samples of soil seed bank of three sites in permanent preservation areas in the region of Rio Negrinho, SC.

\begin{tabular}{lccc}
\hline Variáveis avaliadas & Ambiente I & Ambiente II & Ambiente III \\
\hline Número de sementes germinadas $/ \mathrm{m}^{2}$ & $2846 \mathrm{~A}$ & $4292 \mathrm{~A}$ & $2125 \mathrm{~A}$ \\
Número de espécies registradas & 30 & 28 & 24 \\
Índice de diversidade de Shannon (H') & 3,18 & 3,06 & 2,87 \\
Índice de equabilidade de Pielou (J') & 0,93 & 0,92 & 0,90 \\
\hline
\end{tabular}

Médias seguidas pela mesma letra não diferem entre si pelo teste de Tukey ( $\alpha$ : 5\%; p: 0,50017).

A densidade de sementes viáveis no solo encontrada por outros autores tem apresentado resultados diversos e, em geral, relacionados ao uso anterior, grau de conservação da vegetação natural e bioma onde está inserido. Para vegetação secundária, o número pode alcançar de 3.000 a 8.000 sementes.m² (UHL; CLARK, 1983; GARWOOD, 1989; YOUNG, 1985; BUTLER; CHAZDON, 1998; ARAÚJO et al., 2001). Em áreas de pastagem, Leal Filho (1992) encontrou uma densidade de 2216 sementes $/ \mathrm{m}^{2}$, enquanto Pereira et al. (2010) obtiveram 1896 sementes germinadas $/ \mathrm{m}^{2}$. Por outro lado, em monitoramento de áreas restauradas no interior de São Paulo, Siqueira (2002) observou densidades de 245,87 a 328,53 sementes $/ \mathrm{m}^{2}$. Os resultados encontrados neste trabalho, em áreas sob reflorestamento de Pinus ou onde houve a extração recente de Pinus são semelhantes aos padrões encontrados por outros autores para vegetação secundária.

O número de indivíduos germinados por grupo foi distinto em cada um dos ambientes abordados e mostrou que as arbustivas, as herbáceas e as gramíneas foram os grupos predominantes nos três ambientes estudados. Nos ambientes I (floresta nativa remanescente) e II (extração recente de povoamento de Pinus), destacaram-se os grupos de espécies arbustivas, gramíneas e herbáceas, enquanto no ambiente III (sob povoamento de Pinus) houve predomínio somente do grupo gramíneas (Tabela 3). Esse resultado mostra que o ambiente III, caracterizado pela cobertura com reflorestamento de Pinus, apresenta maiores limitações ao ingresso de espécies no banco de sementes no solo.

O predomínio de espécies arbustivas, herbáceas e gramíneas neste trabalho converge com os resultados apontados por Silva (2008) e com as citações de Monaco et al. (2003). Outros autores (DIAS et al., 2004; SOUZA et al., 2006) também observaram que a elevada ocorrência de espécies herbáceas no banco de sementes dessas áreas pode estar vinculado ao curto ciclo de vida dessas espécies. Em levantamentos realizados em várias regiões tropicais, Garwood (1989) também detectou uma grande proporção de espécies herbáceas presentes no banco de sementes no solo em áreas perturbadas, com valores que variaram entre 25 e $90 \%$ do total de espécies registradas. As espécies herbáceas, em geral, apresentam grande capacidade reprodutiva, pois estão aptas a suportar condições climáticas adversas, 
como é o caso de tolerância a alternâncias de temperaturas e de umidade, além de variações no suprimento de oxigênio (CHRISTOFFOLETI; CAETANO, 1998).

Tabela 3. Densidade de indivíduos $/ \mathrm{m}^{2}$ e por grupo em três ambientes em áreas de preservação permanente na região de Rio Negrinho, SC.

Table 3. Density of individuals $/ \mathrm{m}^{2}$ per group in three environments in areas of permanent preservation in the region of Rio Negrinho, SC.

\begin{tabular}{lccc}
\hline Grupo & Ambiente 1 & Ambiente 2 & Ambiente 3 \\
\hline Arbóreas & $154,0 \mathrm{bcd}$ & $283,5 \mathrm{a}$ & $33,5 \mathrm{a}$ \\
Arbustivas & $896,0 \mathrm{bd}$ & $1008,5 \mathrm{a}$ & $166,5 \mathrm{a}$ \\
Cipós & $29,0 \mathrm{a}$ & $25,0 \mathrm{a}$ & $12,5 \mathrm{a}$ \\
Herbáceas & $771,0 \mathrm{bcd}$ & $1412,5 \mathrm{a}$ & $508,5 \mathrm{a}$ \\
Gramíneas & $929,0 \mathrm{bd}$ & $1500,0 \mathrm{a}$ & $1233,5 \mathrm{~b}$ \\
Pteridófitas & $66,5 \mathrm{bcd}$ & $37,5 \mathrm{a}$ & $41,5 \mathrm{a}$ \\
Pínus & $0,0 \mathrm{ac}$ & $25,0 \mathrm{a}$ & $129,0 \mathrm{a}$ \\
\hline
\end{tabular}

Médias seguidas pela mesma letra nas colunas não diferem entre si pelo teste de Tukey (5\%).

Resultado semelhante foi obtido por Tres et al. (2007) na avaliação do banco de sementes em fazendas produtoras de Pinus taeda no município de Rio Negrinho (SC), que relataram o predomínio de ervas $(47 \%)$ e arbustos $(21 \%)$. Esses resultados, no entanto, diferem de outros estudos realizados nesse bioma. Chami (2008) avaliou o banco de sementes de um fragmento florestal de Floresta Ombrófila Mista da Floresta Nacional (FLONA) de São Francisco de Paula (RS) e obteve 38 espécies herbáceas, 14 arbóreas e 7 arbustivas, enquanto Caldato et al. (1996) avaliaram, por seis meses, o banco de sementes da Reserva Genética de Caçador (SC), onde constataram a presença de 10 espécies arbóreas e 8 arbustivas.

A análise da presença de espécies arbóreas no banco de sementes no solo também evidenciou que esse grupo foi pouco expressivo sob reflorestamento de Pinus, com menos de 10 indivíduos germinados por $\mathrm{m}^{2}$, enquanto que, nas áreas onde houve a extração do reflorestamento, esse valor aumentou para mais de 70 indivíduos por $\mathrm{m}^{2}$, superando os valores encontrados para esse grupo em áreas com vegetação natural remanescente (Figura 2).

A baixa ocorrência de espécies arbóreas no banco de sementes no solo sob reflorestamento de Pinus pode estar relacionada com a presença de dossel, que dificulta a dispersão e a incorporação no solo de sementes de espécies da flora local. No entanto, essa variável deve ser vista com ressalvas, de acordo com Brown (1992). Esse autor relatou que o método de germinação utilizado nesses estudos, apesar de ser o mais utilizado devido à sua facilidade tanto na manipulação quanto no reconhecimento das sementes germinadas, apresenta-se limitado, por subestimar as amostras devido a erros associados à dormência e mortalidade das sementes. No presente trabalho, a maioria das espécies arbóreas que germinou pertence ao grupo ecológico das pioneiras e apresenta dormência.

As espécies que contribuíram com maior quantidade de sementes germinadas no banco de sementes foram Eupatorium compressum, Erechtites valerianifolius e Braquiaria subquadripara, no ambiente I; Eupatorium compressum, Braquiaria subquadripara, Erechtites valerianifolius, Cyperus meyenianus e Mitracarpus hirtus, no ambiente II; Eupatorium compressum, Bulbostylis capillaris, Braquiaria subquadripara, Cyperus meyenianus e Pinus sp. no ambiente III (Tabela 1, Figura 2). Essas espécies representam $25 \%$ da densidade no ambiente I, $41 \%$ no ambiente II e $49,5 \%$ no ambiente III. Os resultados mostrados na figura 2 destacam a densidade de sementes de Sebastiana subquadripara, especialmente nos ambientes II e III, sob a presença de Pinus.

A diversidade, avaliada através do índice de Shannon, mostrou que as três áreas estudadas são semelhantes quanto a esse parâmetro (Tabela 2). No entanto, observou-se uma tendência de diminuição do ambiente I para o III, fato também observado no índice de equabilidade de Pielou. A diversidade mais elevada no banco de sementes no solo em áreas mais conservadas também foi obtida por outros autores, como é o caso de Araújo et al. (2001) na Floresta Amazônica, no Pará, e Pereira et al. (2010) em área inserida no bioma Floresta Estacional Decidual, em Minas Gerais. 


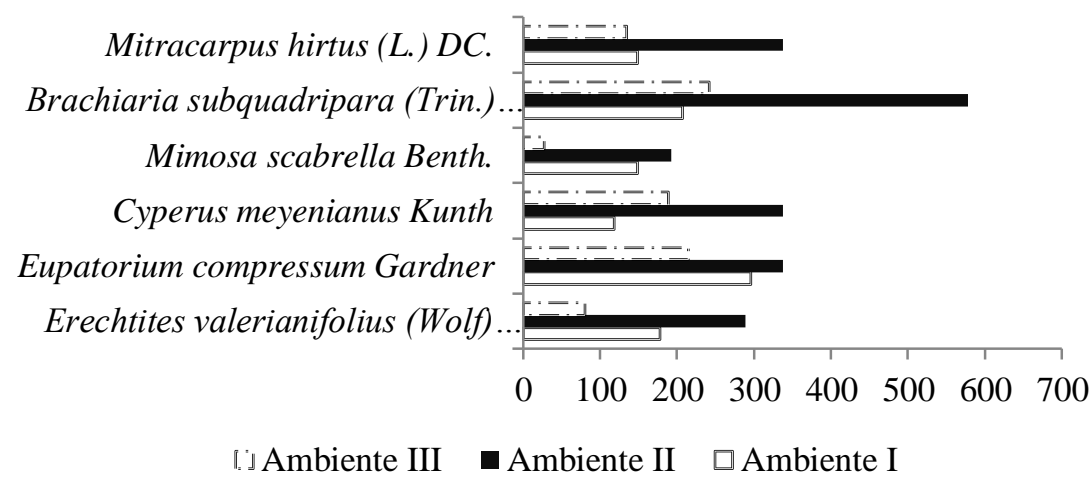

Figura 2. Espécies com maior densidade de sementes germinadas $/ \mathrm{m}^{2}$ nos três ambientes em áreas de preservação permanente na região de Rio Negrinho, SC.

Figure 2. Species with higher seed density in three environments germinate $/ \mathrm{m}^{2}$ in permanent preservation areas in the region of Rio Negrinho, SC.

\section{CONCLUSÕES}

De acordo com os resultados obtidos no presente trabalho, é possível concluir que:

- O banco de sementes no solo após extração recente de Pinus apresentou maior densidade de indivíduos $/ \mathrm{m}^{2}$.

- O tipo de uso e cobertura da área tem relação com a densidade dos grupos de plantas presentes no banco de sementes no solo. No banco de sementes no solo em área sob reflorestamento de Pinus, somente as gramíneas apresentaram superioridade em densidade. Em áreas sob floresta nativa remanescente, o banco de sementes apresentou maior densidade de espécies arbustivas e gramíneas.

- O número de espécies e a diversidade foram decrescentes do ambiente com floresta nativa remanescente para o ambiente sob reflorestamento de Pinus.

- O banco de sementes no solo nos ambientes I e II possui melhor potencial para a recuperação de áreas degradadas, considerando a quantidade de propágulos presentes e a diversidade de espécies e formas de vida.

\section{REFERÊNCIAS}

ARAÚJO, M. M.; OLIVEIRA, F. A.; VIEIRA, I. C. G.; BARROS, P. L. C.; LIMA, C. A. T. Densidade e composição florística do banco de sementes do solo de florestas sucessionais na região do Baixo Rio Guamá, Amazônia Oriental. Scientia Forestalis, Piracicaba, v. 59, p. 115 - 130, 2001.

BAIDER, C.; TABARELLI, M; MANTOVANI, W. O banco de sementes de um trecho de Floresta Atlântica Montana (São Paulo, Brasil). Revista Brasileira de Biologia, Recife, v. 2, n. 59, p. 319 - 328 , 1999.

BROKAW, N.VL. Treefalls, regrowth and community structure in tropical forests. In: PICKETT, S. T. A.;WHITE, P. S. (eds.). The ecology of natural disturbance and patch dynamics. San Diego: Academic Press, 1985. p. 53 - 69.

BROWER, J. E.; ZAR, J. H. Field and laboratory methods for general ecology. Boston: W. C. Brown Publishers, 1984. 226 p.

BROWN, D. Estimating the composition of a forest seed bank: a comparison of the seed extraction and seedling emergence methods. Canadian Journal of Botany, v. 70, n. 8, p. 1603 - 1612, 1992.

BUTLER, B. J.; CHAZDON, R. L. Species richness, spatial variation and abundance of soil seed bank of a secondary tropical rain forest. Biotropica, Storrs, v. 30, n. 2, p. 214 - 222, 1998.

CALDATO, S.; FLOOS, P. A.; CROCE, D. M.; LONGHI, S. J. Estudo da regeneração natural, banco de 
sementes e chuva de sementes na reserva genética florestal de Caçador, SC. Ciência Florestal, Santa Maria, v. 6, n. 1, p. 27 - 38, 1996.

CHAMI, L. B. Vegetação e mecanismos de regeneração natural em diferentes ambientes da Floresta Ombrófila Mista na FLONA de São Francisco de Paula, RS. 121 f. Dissertação (Mestrado em Engenharia Florestal) - Universidade Federal de Santa Maria, Santa Maria, 2008.

CHRistoffoleti, P. J.; CAETANO, R. S. X. Soil seed banks. Scientia Agrícola, v. 55, p. 74 - 78, 1998.

DANIEL, O.; JANKAUSKIS, J. Avaliação de metodologia para o estudo do estoque de sementes do solo. Revista IPEF (Atual Scientia Forestalis), Piracicaba, v. 41 - 42, p. 18 - 26, 1989.

DIAS, H. K. O.; ARAÚJO, M. M.; MODESTO, E. L. OLIVEIRA, J. N. de. Densidade e composição florística do banco de sementes do solo em floresta secundária na Amazônia Oriental. In: SIMPÓSIO LATINO-AMERICANO SOBRE MANEJO FLORESTAL, 3., 2004, Santa Maria. Anais... Santa Maria: UFSM, Programa de Pós-Graduação em Engenharia Florestal, 2004. p. 226 - 232.

GABINETE DE PLANEJAMENTO DO ESTADO DE SANTA CATARINA (GAPLAN). Atlas de Santa Catarina. Aerofoto Cruzeiro. Rio de Janeiro. 1986. 173 p.

GARWOOD, N. C. Tropical soil seed banks: a review. In: LECK, M. A.; PARKER, V. T.; SIMPSON, R. L. (ed.). Ecology of soil seed banks. London: Academic Press, 1989. p. 149 - 209.

HALL, J. B.; SWAINE, M. D. Seed Stocks in Ghanaian Forest Soils. Biotropica, Legon, v. 12, p. 256 263, 1980.

HARPER, J. L. Population biology of plants. New York: Academic Press, 1977. 892 p.

KOBIYAMA, M.; CHECCHIA, T.; CORSEUIL, C. W.; LINO, J. F. L.; LOPES, N. H. Y.; GRISON, F.; CHAFFE, P. L. B.; MALUTTA, S.; RIBAS JÚNIOR, U.; LANGA, R.; BASSO, S. Forest hydrology Project (UFSM-MOBASA) for water management in Rio Negrinho City, Santa Catarina, Brazil. In: VAN de GIESEN, XIA, J.; ROSBJERG, D.; FUKUSHIMA, Y. (eds.). Changes in Water Resources Systems: Methodologies to maintain water security and ensure integrated management. Wellington: IAHS. 2007. p. 250 - 257. (IAHS Publication 315).

LEAL FILHO, N. Caracterização do banco de sementes de três estádios de uma sucessão vegetal na Zona da Mata de Minas Gerais. 116 f. Dissertação (Mestrado em Ciência Florestal) - Universidade Federal de Viçosa, Viçosa, 1992.

MARTINEZ- RAMOS, M.; SOTO-CASTRO, A. Seed rain and advanced regeneration in a tropical rain Forest. In: FLEMING, A.; ESTRADA, A. (eds.). Frugivory and seed dispersal: ecological and evolutionary aspects. Dordrecht: Kluwer Academic Pulishers, p. 299 - 318, 1993.

MARTINS, S. V.; RODRIGUES, R. R.; GANDOLFI, S.; CALEGARI, L. Sucessão ecológica: fundamentos e aplicações na restauração de ecossistemas florestais. In: MARTINS, S. V. (ed.). Ecologia de Florestas Tropicais do Brasil. Viçosa: Editora UFV. 2009, 61 p.

MARTINS, S. V. Recuperação de áreas degradadas: ações em áreas de preservação permanente, voçorocas, taludes rodoviários e de mineração. Viçosa, MG: Editora Centro de Produções Técnicas, 2009. 270 p.

MONACO, L. M.; MESQUITA, R. C. G.; WILLIAMSON, G. B. Banco de sementes de uma floresta secundária amazônica dominada por Vismia. Acta Amazonica, Manaus, v. 33, n. 1, p. 41 - 52, 2003.

MURDOCH, A. J.; ELLIS, R. H. Longevity, viability and dormancy. In: FENNER, M. Seeds: the ecology of regeneration in plant communities. CAB International, Wallingford, U.K. 1992, p. 193 - 229.

OLIVEIRA, E. C. Morfologia das plântulas. In: AGUIAR, I. B.; PIÑA RODRIGUES, F. C. M.; FIGLIOLIA, M. B. Sementes florestais tropicais. Brasília: ABRATES, 1993, p. 175 - 211.

PARKER, V. T.; SIMPSON, R. L.; LECK, M. A. Pattern and process in the dynamics of seed banks. In: LECK, M. A.; PARKER, V. T.; SIMPSON, R. L (eds.). Ecology of soil seed banks. New York: Academic Press, 1989, p. 367 - 384. 
PEREIRA, I. M.; ALVARENGA, A. P.; BOTELHO, S. A. Banco de sementes do solo, como subsídio à recomposição de mata ciliar. Floresta, Curitiba, v. 40, n. 4, p. 721 - 730, 2010.

PINÂ-RODRIGUES, F. C. M.; COSTA, L. G. S.; REIS, A. Estratégias de estabelecimento de espécies arbóreas e o manejo de florestas tropicais. In: CONGRESSO FLORESTAL BRASILEIRO, 6, 1990, Campos do Jordão. Anais... Campos do Jordão: Sociedade Brasileira de Silvicultura, 1990, p. 676 - 683.

PUTZ, F. E. Treefall pits and mounds, buried seeds, and importance of soil disturbance to pioneer trees on Barro Colorado Island, Panamá. Ecology, Gainesville, v. 64, n. 5, p. 1069 - 1074, 1983.

SCHMITZ, M. C. Banco de sementes no solo em áreas do reservatório da UHE Paraibuna. In: KAGEYAMA, P. Y. Recomposição da vegetação com espécies arbóreas nativas em reservatórios de usinas hidrelétricas da CESP. SÉRIE IPEF, Piracicaba, v. 8, n. 25, p. 7 - 8. 1992.

SILVA, A. J. C. Banco de sementes no solo de planícies inundáveis inseridas na Floresta Ombrófila Mista no Estado do Paraná. 80 f. Dissertação (Mestrado em Engenharia Florestal) - Universidade Federal do Paraná, Curitiba, 2008.

SIMPSON, R. L; LECK, M. A.; PARKER, V. T. Ecology of soil seed banks. California: Academic Press, 1989. $385 \mathrm{p}$.

SIQUEIRA, L. P. de. Monitoramento de áreas restauradas no interior do estado de São Paulo, Brasil. 116 f. Dissertação (Mestrado em Conservação e Ecossistemas Florestais) - Escola Superior de Agricultura Luiz de Queiroz, Piracicaba, 2002.

SORREANO, M. C. M. Avaliação de aspectos da dinâmica de florestas restauradas, com diferentes idades. 145 f. Dissertação (Mestrado em Engenharia Florestal) - Escola Superior de Agricultura Luiz de Queiroz, Piracicaba, 2002.

SOUZA, M. L. de. Análise do banco de sementes no solo e da regeneração natural de um fragmento florestal com Araucaria angustifolia (Bert.) O. Ktze., no estado do Paraná. 115 f. Dissertação (Mestrado em Ciências Florestais) - Universidade Federal do Paraná, Curitiba, 1996.

SOUZA, P. A.; VENTURIN, N.; GRIFFFITH, J. J.; MARTINS, S. V. Avaliação do banco de sementes contido na serapilheira de um fragmento florestal visando recuperação de áreas degradadas. Cerne, Lavras, v. 12, n. 1, p. 56 - 67, 2006.

TRES, D. R.; SANT'ANNA, C. S.; LANGA, S. B. R.; RIBAS JÚNIOR, U.; REIS, A. Banco e chuva de sementes como indicadores para a restauração ecológica de matas ciliares. Revista Brasileira de Biociências, Porto Alegre. v. 5. p. 309 - 311, 2007.

UHL, C.; CLARK, H. Seed ecology of selected amazon basin sucessional species. Botanical Gazette, v. 144 , p. $419-425,1983$.

UHL, C.; BUSCHBACHER, R.; SERRÃO, E. A. S. Abandoned pastures in Eastern Amazonia: 1-patterns of plants succession. Journal of Ecology, Athens, v. 76, p. 663 - 681, 1988.

VIANA, V. M. Seed and seedling availability as a basis for management of natural forest regeneration. In: ANDERSON, A. B. Alternatives to deforestation: steps toward sustainable use of the Amazon Rain Forest. Columbia University Press, New York, 1990, p. 99 - 115.

VIEIRA, N. K.; REIS, A. O papel do banco de sementes na restauração de áreas degradadas. Disponível em: <http://www.sobrade.com.br/eventos/2003/seminario/Trabalhos/028.pdf>. Acesso em: 15/03/2011.

WHITMORE, T. C. Gaps in the Forest canopy. In: TOMLINSON, P. B.; ZIMMERMAN, M. H. (eds.). Tropical trees as living systems. Cambridge University Press, Cambridge. 1978. p. 639 - 655.

A review of some aspects of tropical rain forest seedling ecology with suggestion for further enquiry. In: SWAINE, M. D. (ed.). The ecology of tropical forest tree seedling. Paris: UNESCO; The Pathernon Publishing Group, Paris, 1996. p. 3 - 39.

YOUNG, K. R. Deeply buried seeds in a tropical wet forest in Costa Rica. Biotropica, Gainesville, v. 17, n. 4. p. $336-338,1985$. 